Michael Steen

Klinik für Plastische und Handchirurgie, Brandverletztenzentrum, BG-Kliniken Bergmannstrost, Halle

\title{
Viertgradige Verbrennung des Kniegelenks beim Schwerstverbrannten
}

\section{Fallbeispiel}

\section{Falldarstellung}

Ausgedehnte Brandverletzungen mit freiliegendem Knochen, Sehnenverlusten und Gelenkeröffnung stellen eine Indikation für die primäre Defektdeckung und frühzeitige Rekonstruktion dar. Der dargestellte Patient verlor das Lig. patellae mit der angrenzenden Kortikalis der Patella und der Tuberositas tibiae im Rahmen ausgedehnter weiterer Verletzungen. Eine gefäßgestielte Muskellappenplastik aus dem medialen und lateralen M. gastrocnemius war in der Lage, eine Infektion und die weitere Nekrose zu verhindern. Unter der gut durchbluteten Lappenplastik wurde dann sekundär nach 9 Monaten die Rekonstruktion des Lig. patellae mit einer aufgeflochtenen Sehne des M.tibialis posterior vorgenommen. Die Verankerung erfolgte durch Aufschrauben von Leiterplatten aus dem Profile-Set ${ }^{\circledR}$ für die Handchirurgie (Fa. Leibinger). Der Patellahochstand konnte nicht mehr ausgeglichen werden. Die Revaskularisierung der Sehnentransplantate wurde mittels MRT kontrolliert. Nach 8 Wochen wurde zunehmend belastet, nach 16 Wochen konnte Gehfähigkeit auf Treppen ohne Gehhilfe erreicht werden.

\section{Schlüsselwörter}

Autologe Rekonstruktion des Lig. patellae . Schwerbrandverletzte · Autologe Sehnentransplantation
Ein zum Zeitpunkt des Unfalls 59-jähriger Mann wurde bei einem Kfz-Unfall eingeklemmt und erlitt zweit- und drittgradige Verbrennungen von $38 \%$ der Körperoberfläche. Betroffen waren Kopf, Rumpf, linkes Bein, beide Arme und Hände. Areale im Bereich des linken Knies, der rechten Hand einschließlich Unterarm, der linken Hand sowie der Stirn waren unter Mitbeteiligung des Skeletts tief verbrannt. Komplizierend lagen ein Inhalationstrauma und ein Schädel-Hirn-Trauma jeweils 2. Grads vor.

Während des mehrmonatigen, teilweise komplizierten intensivmedizinischen Verlaufs musste die rechte Hand im Unterarm amputiert werden. An der linken Hand verlor der Verletzte die Finger 4 und 5, die übrigen Anteile der Hand konnten bei Verlust des Streckapparats und der dorsalen Gelenkkapseln mit einem Leistenlappen erhalten werden. Bei erhaltener Sensibilität konnte so ein funktionsfähiger Spitz- und Schlüsselgriff erreicht werden. An der Stirn war die Spalthauttransplantation nach Abtragung der Tabula externa möglich.

\section{Kniebefund}

Bei der Abtragung der Nekrosen am linken Bein fand sich das Lig. patellae avaskulär durch die Hitzeeinwirkung, die streckseitige Kortikalis der Patella war verkohlt, ebenso der Übergang auf die Tuberositas tibiae. Das Kniegelenk war nach Entfernung des nekrotischen Gewebes eröffnet (Abb. 1).

\section{Primärversorgung des Knies}

Zur sofortigen Defektdeckung im Rahmen der Nekrosektomie wurden 2 gefäßgestielte Lappenplastiken aus dem medialen und lateralen $M$. gastrocnemius verwendet (Abb. 2). Die beiden Lappenplastiken wurden über dem Kniegelenk versetzt gegeneinander mit resorbierbaren Nähten fixiert und deckten die gesamte freiliegende Knochenoberfläche und das eröffnete Kniegelenk ab. Die Umgebung der Lappenplastiken am Ober- und Unterschenkel war bis auf die Faszie abgetragen worden. Die gesamte Oberfläche der transferierten Muskeln und die angrenzenden Faszien am Ober- und Unterschenkel wurden temporär mit synthetischem Hautersatz (Lyomousse ${ }^{\circledR}$ ) abgedeckt.

Nach etwas mehr als 1 Woche war der Übergang zwischen den Muskelanteilen und auf die Faszien stabil genug und kein Hinweis auf eine tiefe Infektion zu finden, sodass die Deckung mit Mesh-graft-Transplantaten erfolgen konnte (Abb. 3).

\footnotetext{
๑) Springer-Verlag 2003

Priv.-Doz.Dr. Michael Steen

Klinik für Plastische und Handchirurgie,

Brandverletztenzentrum,

BG-Kliniken Bergmannstrost,

Merseburger Straße 165, 06112 Halle,

E-Mail:michael.steen@bergmannstrost.com,

Tel.:0345-1326333, Fax:0345-1326334
} 


\section{Steen \\ Deep burn of the knee joint. Case report}

\begin{abstract}
Extensive burn injuries accompanied by exposed bone, loss of tendon and traumatic arthrotomy are indications for primary closure of defects and early reconstruction. 0 wing to major burn injury, the patient presented in this paper suffered loss of the patellar ligament including the adjacent cortical substance of the patella and the tuberosity of the tibia. Gauntlet flaps from the medial and lateral gastrocnemius muscle made it possible to prevent further necrosis and avoid infection. The flaps had a good blood supply, and 9 months later a plaited tendon of the posterior tibialis muscle was used to reconstruct the patellar ligament under the flaps as a secondary procedure. It was fixed by screwing on ladder plates selected from the Profile-Set range manufactured by Leibinger for use in hand surgery. It was not possible to correct the cranial malposition of the patella. Revascularization of the transplanted tendon was controlled by MR tomography. After 8 weeks weightbearing was increased, and after 16 weeks the patient was again able to climb stairs without walking aids.
\end{abstract}

\section{Keywords}

Autologous reconstruction of the patellar ligament $\cdot$ Major burn injury · Autologous tendon transplantation

\section{Abb. 1 Tiefe Verbrennung der Kniescheibe und Nekrose des Lig. patellae - intraoperativer Befund während der Nekrosektomie}

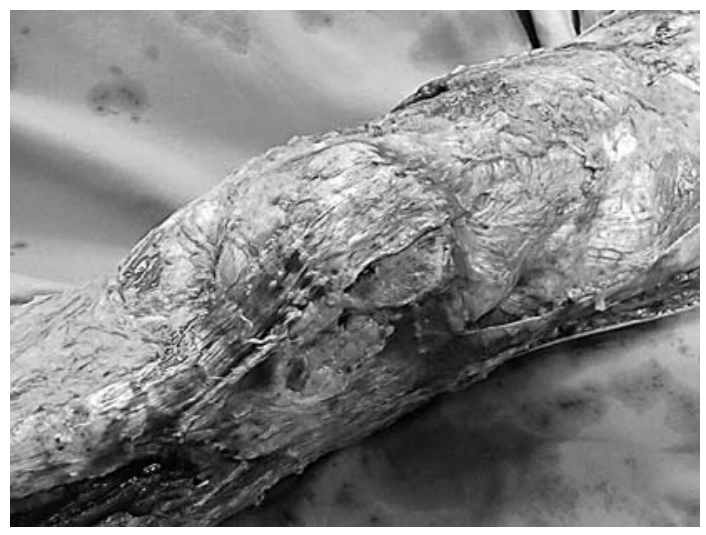

Abb. 2 Präparation des medialen und lateralen gefäßgestielten M. gastrocnemius

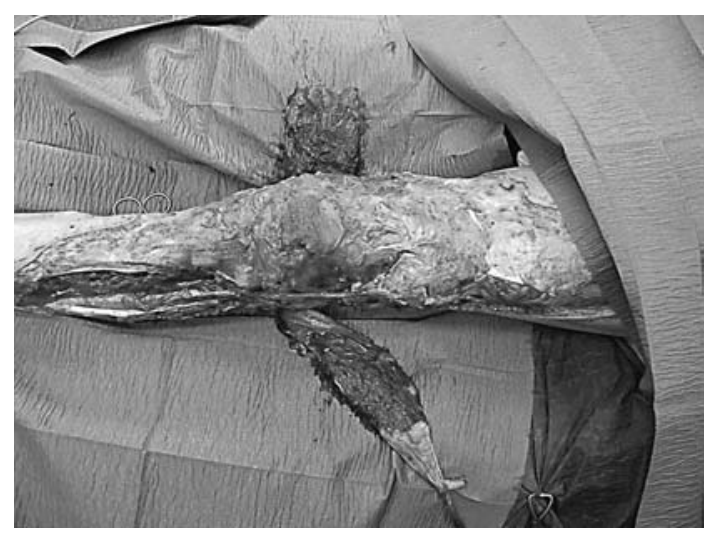

Eine primäre Rekonstruktion des Lig. patellae erfolgte nicht. Dies begründete sich darauf, dass zu diesem Zeitpunkt die Knochenoberflächen an der Tuberositas tibiae und der Patella nach der Kortikalisabtragung für eine Verankerung nicht geeignet erschienen. $\mathrm{Zu}$ dem waren aufgrund der Komplexität der Verletzungen und den evtl. notwendigen Rekonstruktionen auch an den oberen Extremitäten die zur Verfügung stehenden Spenderbereiche noch nicht einzuschätzen.

\section{Rehabilitation}

Der Verletzte überlebte und wurde von der Brandverletztenintensivstation in eine Rehabilitationseinrichtung verlegt. Im Vordergrund stand die Gewinnung ausreichender Funktion der oberen Extremitäten einschließlich der Prothesenversorgung. Der Verletzte erlangte schrittweise eine ausreichende Selbstständigkeit, sodass im Verlauf der Rehabilitation 9 Monate nach dem Unfall die Rekonstruktion des Lig. patellae erfolgte.
Abb. 3 Verschluss des Kniegelenks mit den Muskellappen

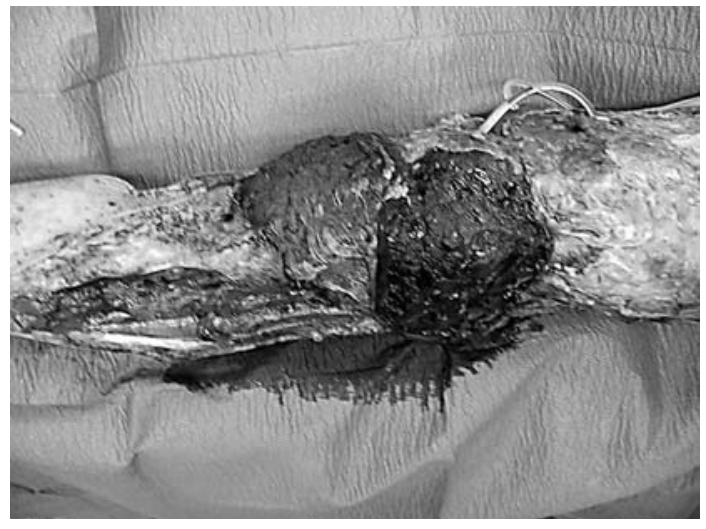




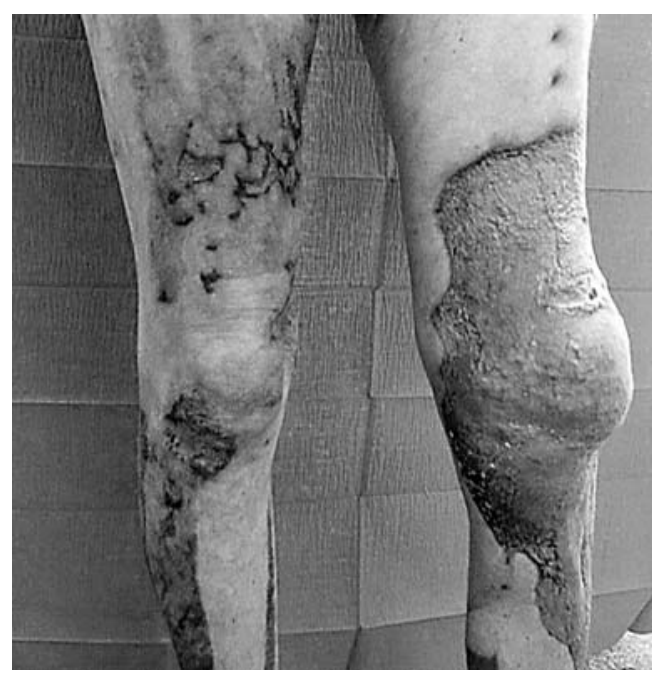

Abb. $4<$ Frühergebnis nach Einheilung der Muskellappen und Spalthauttransplantation während der weiteren Rehabilitation
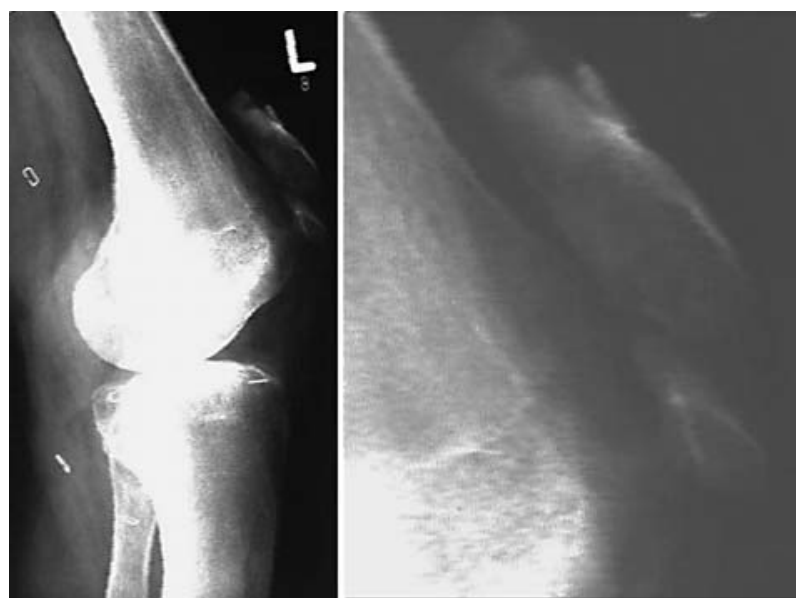

Abb. $5<$ Seitliches Röntgenbild des linken Kniegelenks aufgehellt und mit Vergrößerung der Patella

\section{Rekonstruktion des Lig. patellae}

Zur Rekonstruktion des Lig. patellae wurden die beiden Muskellappen über dem Knie auf ihren Grenzen umschnitten und angehoben. Die Tuberositas tibiae und die nach kranial verlagerte $\mathrm{Pa}$ tella wurden dargestellt. Die Knochenoberflächen fanden sich glatt und verfestigt im Sinn der Ausbildung einer Neokortikalis (Abb. 4).

Die Wahl zwischen allogenen und autologen Transplantaten wurde zugunsten eines autologen Transplantats entschieden. Als Material für den Ersatz des Lig. patellae links kam bei diesem Patienten nur Gewebe aus dem rechten Bein in Frage. Zwischen der Entnahme eines Drittels der rechten Patellarsehne, eines Anteils Fascia lata oder einer Sehne des M. tibialis posterior fiel die Entscheidung im Zusammenwirken mit dem Verletzten auf die Tibialis-posterior-Sehne, von deren Entnahme die ge- ringsten Risiken für die einzige unverletzte Extremität erwartet wurden $[1,5$, 9].

Die kräftige Sehne wurde 3 fach gelegt und verflochten. Durch die Verdrehung und das Flechten ist eine Steigerung der Stabilität zu erwarten [7]. Die
Verankerung auf der Tibia und auf der Patella erfolgte mit 2 4-Loch-Titan-Leiterplatten und 1 6-Loch-Titan-Leiterplatte aus dem Profile-Set ${ }^{\circledR}$ für die Handchirurgie (Fa. Leibinger). Die Sehnenanteile wurden dazu unter den quer gelegten Leiterplatten hindurchgezogen und beim Festschrauben der Platten auf den Knochen fixiert. Ein Nachgeben wie bei einer Nahtverankerung wurde damit verhindert [6]. Es gelang wegen der Schrumpfung des M. quadriceps nicht, die Patella wieder vollständig in ihre alte Position zu bringen. Aufgrund der Weichteilverhältnisse nach epifaszialer Transplantation in der Umgebung wurde auf eine Distraktion verzichtet [2]. Eine Sicherung der Transplantate erfolgte zusätzlich durch eine Zuggurtung, welche in der Tibia über eine quer eingebrachte kanülierte Schraube und am Oberrand der Patella unter der Quadrizepssehne geführt wurde. Die Muskellappen wurden dann über den Sehnentransplantaten wieder zurückverlagert und verschlossen (Abb. 5, Abb. 6).

\section{Belastung nach Rekonstruktion}

Die übertragenen Sehnen heilten komplikationslos ein. Nach 2 Wochen wurde eine limitierte Bewegung mit einer Motorschiene begonnen, welche langsam gesteigert wurde. Da Sehnengewebe verwendet wurde, waren im Kern der Sehnen eine verzögerte Revaskularisierung und ein spätes Erreichen der Stabilität zu erwarten [3]. Eine Belastung wurde deshalb erst nach dem Nachweis der sicherer Revaskularisierung im MRT zugelassen.

Die Zuggurtung der Stichinzisionen wurde nach 8 Wochen entfernt. Die Re-
Abb. 6 Röntgenbild des linken Kniegelenks in 2 Ebenen nach Abschluss der Rekonstruktion des Lig. patellae. Die Fixierung der Sehnenenden mit den Titanleiterplatten aus dem Profile-Set ${ }^{\circledR}$ für Handchirurgie bleiben in situ
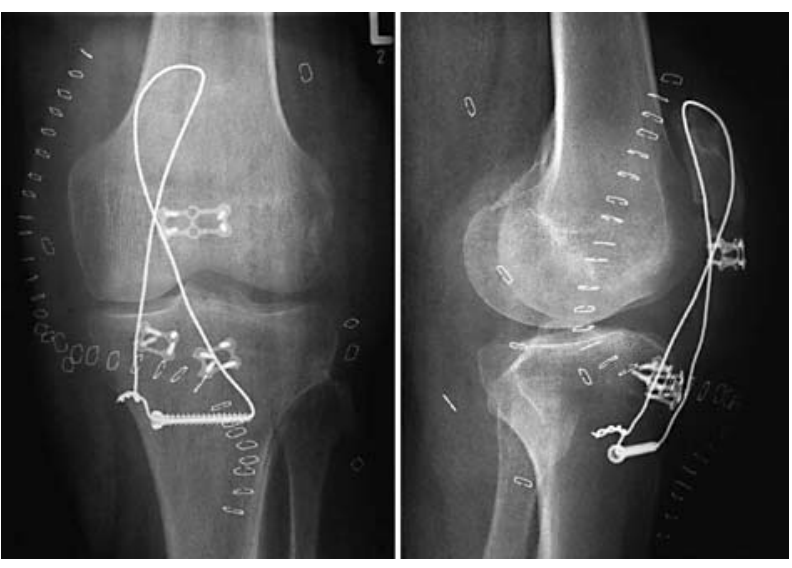

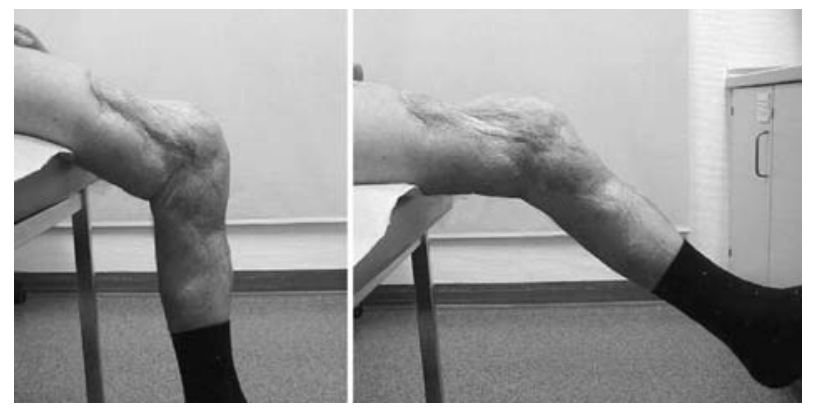

Abb. $7<$ Aktive Streckung des Knies gegen die Schwerkraft
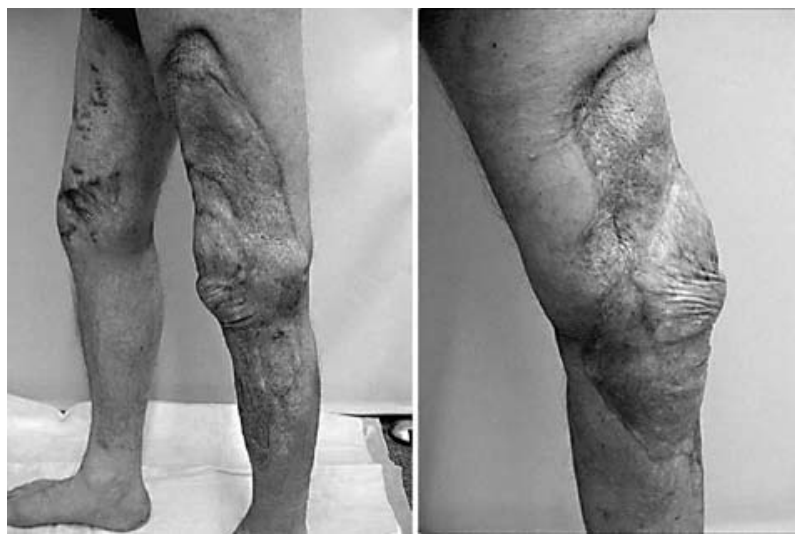

Abb. $8<$ Lokalbefund im Bereich des linken Knies nach Rehabilitation

vaskularisierung der Sehnentransplantate wurde mittels MRT nach 8 und 16 Wochen verfolgt [8]. Nachdem eine Durchblutung nachgewiesen werden konnte, erfolgte beginnend 8 Wochen nach dem Sehnentransfer ein weiterer aktiver Belastungsaufbau, welcher nach 16 Wochen mit freier Gehfähigkeit auch auf Treppen beendet werden konnte. $\mathrm{Zu}$ diesem Zeitpunkt waren eine Streckung und Beugung von $0-25-75^{\circ}$ erreicht (Abb. 7, Abb. 8).

\section{Nachbeobachtungszeit und bisherige Ergebnisse}

Die Nachbeobachtungszeit betrug bis zum Zeitpunkt der Beitragserstellung 1 Jahr und 9 Monate seit Behandlungsabschluss. Bei der autologen Rekonstruktion des vorderen Kreuzbandes mit einem Drittel der gleichseitigen Patellarsehne sind Einzelfälle von Spätrupturen des geschwächten Lig. patellae bis zu 6 Jahre nach dem Eingriff beobachtet worden [4]. Es bleibt daher nach knapp 2 Jahren abzuwarten, ob die Rekonstruktion langfristig stabil bleibt.

\section{Schlussfolgerungen}

Bei ausgedehnten Brandverletzungen mit freiliegendem Knochen, Sehnenverlusten und Gelenkeröffnung sind frühzeitige Lappenplastiken in der Lage, Infektion und weitere Nekrose zu verhindern. Damit werden Funktionserhalt und weitere rekonstruktive Operationen ermöglicht. Unter gut durchbluteten Lappenplastiken sind auch Sehnenrekonstruktionen kräftiger und stark belasteter Sehnen wie des Lig. patellae möglich.

Das aufgezeigte Vorgehen kann als eine gangbare Möglichkeit der sekundären Rekonstruktion der Patellarsehne bewertet werden. Bei isolierten Verletzungen des Knies und günstiger Verteilung der Gesamtverletzung sollte die Rekonstruktion primär zusammen mit der Muskellappenplastik durchgeführt werden, um eine Schrumpfung des M. quadriceps zu verhindern.

\section{Literatur}

1. Falconiero RP, Pallis MP (1996) Chronic rupture of a patellar tendon: a technique for reconstruction with Achilles allograft. Arthroscopy 12:623-626

2. Isiklar ZU, Varner KE, Lindsey RW, Bocell JR, Lintner DM (1996) Late reconstruction of patellar ligament ruptures using llizarov external fixation.Clin Orthop 322:174-178

3. Kuhlmann JN, Luboinski J, Mimoun M, Orcel L, Baux S (1994) Reconstruction du ligament collateral lateral du genou du rat par un transplant libre autogene constitue de fascia lata, de ligament ou de tendon. Acta Orthop Belg 60:10-18

4. Marumoto JM, Mitsunaga MM, Richardson AB, Medoff RJ, Mayfield GW (1996) Late patellar tendon ruptures after removal of the central third for anterior cruciate ligament reconstruction. A report of two cases. Am J Sports Med 24:698-701

5. Müller B, Rupp S, Kohn D, Seil R (2000) Entnahmestellenproblematik nach vorderer Kreuzbandplastik mit dem mittleren Drittel der Patellarsehne. Unfallchirurg 103:662-667

6. Nakano H, Yasuda K, Tohyama H, Yamanaka M, Wada T, Kaneda K (2000) Interference screw fixation of doubled flexor tendon graft in anterior cruciate ligament reconstruction - biomechanical evaluation with cyclic elongation. Clin Biomech 15: 188-195

7. Thambyah A, Thiagarajan P, Goh JC (2000) Biomechanical study on the effect of twisted human patellar tendon. Clin Biomech 15:756760

8. Tosch U, Sander B, Schubeus P, Lais E, Hertel P, Felix R (1990) Kernspintomographische Verlaufsbeurteilung der vorderen Kreuzbandersatzplastik mit einem autologen Ligamentumpatellae-Transplantat. Rofo Fortschr Geb Rontgenstr Neuen Bildgeb Verfahr 153:716-720

9. Weber K, Schmidgen A, Wentzensen A (2000) Patellarsehnenruptur nach vorderer Kreuzbandrekonstruktion mit autologem Patellarsehnen-Knochen-Transplantat - eine Kasuistik.Unfallchirurg 103:1124-1127 